\title{
LA NOVELA DE MI VIDA: JUGANDO CON LA MEMORIA
}

\author{
Jan Mlčoch \\ Universidad de Ostrava \\ https://doi.org/10.18778/8220-195-6.24
}

\section{Resumen}

La posmodernidad ha permitido borrar las fronteras entre la realidad y la ficción. En estas circunstancias apareció la nueva novela histórica que tenía por objetivo contribuir al debate sobre la memoria colectiva. El arte - en nuestro caso la literatura- ha reemplazado a la historia como fuente del saber histórico. La novela de mi vida (2002), con su peculiar estructura metaficcional, del autor cubano Leonardo Padura nos permite hacer un análisis literario a través del cual comentamos los límites borrosos entre la ficción y la realidad haciendo hincapié en la construcción de la memoria histórica de los cubanos.

Palabras clave: Leonardo Padura, metaficción, memoria histórica, Cuba.

En las últimas décadas somos testigos en el área de las humanidades del prolífero interés por el pasado. En el año 1979 dicta Alejo Carpentier la conferencia en la Universidad de Yale titulada "La novela latinoamericana en vísperas de un nuevo siglo", en la cual proclama que el deber del escritor hispanoamericano es dar cuenta de lo que pasa a su alrededor y convertir así su narrativa en la "nueva crónica de Indias". La obra del novelista cubano demuestra -junto con muchísimos ejemplos más- el interés por la historia que en la literatura hispanoamericana cobra una especial relevancia porque prácticamente desde sus obras inaugurales -como son las cartas, crónicas, diarios de los navegantes y conquistadores- esta literatura combina en sí tanto la ficción como la historiografía. González Echeverría (2011) habla de "ficciones del 
archivo", es decir, "un tipo de novela que dialoga con la historia, generalmente ubicada en época colonial" (Viu, 2007: 174). En estos textos la cantidad de información histórica proporcionada es asombrosa y hacen de la lectura no solo una actividad placentera sino también un proceso educativo. Esta "nueva novela hispanoamericana" ha visto su continuación también en los textos escritos bajo el paradigma del posmodernismo.

Según Linda Hutcheon, uno de los rasgos definitorios del posmodernismo es la importancia de la metaficción historiográfica (Hutcheon, 2014). El privilegio de saber contar lo ocurrido que hasta entonces había sido reservado a los trabajos historiográficos se desintegró en una multitud de voces que proclamaban su punto de vista, decían su versión de lo ocurrido e incluso insistían en la veracidad de lo contado. La literatura ha ocupado pues el papel de la historiografía. Esta multiplicación de voces se debe también a otro hecho clave que podemos ver como generacional: mientras que la generación del boom creía todavía en un proyecto en común ${ }^{1}$, los escritores más jóvenes dejaron de creer en una sola verdad, una verdad absoluta y totalizadora. Esta imposibilidad de encontrar una visión conjunta se ha transformado en una fragmentación total de la sociedad occidental que vivimos hoy en día. Hay que destacar también que esta fragmentación ha imposibilitado crear una utopía colectiva similar a la del comunismo del siglo pasado que tuvo, junto con el nazismo, consecuencias desastrosas. Esta desventaja en pro del futuro representa una de las razones por las cuales el mundo de hoy se vuelve al análisis cada vez más profundo del pasado. En las últimas décadas ha irrumpido en la teoría literaria el concepto de la "memoria histórica" que otorga un valor historiográfico al relato individual, convirtiéndolo así en una herencia colectiva grabada en la memoria de dicha colectividad. La cantidad de obras que se han publicado en Argentina, Chile o España - por poner algún ejemplo- muestra no solo el interés por parte de los lectores, sino, sobre todo, la necesidad de estas tres naciones de repensar su propio pasado, contrastando

1 Podemos en este lugar destacar que la absoluta mayoría de los escritores del boom eran marxistas declarados. 
versiones peculiares reproducidas por las creaciones artísticas y la versión oficial. Sería redundante detenernos en una profundización del concepto ya que existe una riquísima bibliografía al respecto, pero mencionemos al menos que mientras que en un principio había un trauma que se suponía mitigar con el relato literario, hoy en día este arte se ha convertido de nuevo en un arma política, que en muchos casos pretende justificar el fracaso de las utopías colectivas antes mencionadas.

Es precisamente este análisis del pasado que también está detrás de La novela de mi vida del escritor cubano Leonardo Padura. Para formular nuestras observaciones, partamos del siguiente comentario escrito por el propio autor en una especie de prólogo a la primera edición:

Aunque sustentada en hechos históricos verificables y apoyada incluso textualmente por cartas y documentos personales, la novela [...] debe asumirse como obra de ficción (Padura, 2002a: 11).

Esta cita contradictoria nos muestra dos palabras clave que como dos hilos conductores formarán el tejido de nuestro comentario: hechos históricos, por un lado, y ficción, por otro. Ya Aristóteles en su Poética distinguía entre la Historia y la Poesía, reservando a la segunda la capacidad y, sobre todo, la posibilidad y legitimidad de "engañar", por ello nos atrevemos a buscar los borrosos límites entre la historia y la ficción en La novela de mi vida de Leonardo Padura.

Para una mejor comprensión suponemos necesario presentar un par de datos clave. La novela, publicada en 2002 por la editorial Tusquets, narra una historia de un tal Fernando Terry. Este personaje es un exiliado cubano que vive en Madrid y que recibe un permiso por parte del gobierno cubano para volver a la isla durante quince días. El principal motivo de su regreso fue la necesidad de encontrar un texto escrito por José María Heredia, poeta romántico, que supuestamente dictó Heredia en su lecho de muerte. Durante estos quince días Terry se encuentra con su madre, con sus amigos -durante sus estudios universitarios pertenecía a un 
grupo artístico llamado "Los socarrones"- con su amor platónico y con profesores de la universidad donde escribía tesis de doctorado precisamente sobre Heredia y de la cual fue botado por una maniobra de la policía secreta de Cuba. Durante todos estos encuentros, a Terry se le abren heridas que dejó sin cicatrizar, sobre todo su convencimiento de que alguno de sus amigos íntimos le había delatado a la policía. La búsqueda de este "culpable" es una constante en los pensamientos del protagonista hasta el final de la novela. Sin embargo, pese a posibles rencores, Terry y sus amigos emprendieron una búsqueda incesante de este manuscrito perdido de Heredia que podría tener un valor único y singular para los cubanos. Viajan a todos los lugares que pudieran tener alguna relación con Heredia, sobre todo, a las antiguas logias masónicas que tan importante papel jugaron a principios del siglo XIX en el proceso de emancipación cubano. Esta narración (A) es solamente una de las tres tramas que forman el conjunto de la novela.

La segunda (B) se refiere a la vida del manuscrito escrito por Heredia, que, al principio, está en manos de José de Jesús de Heredia, el hijo menor del poeta, que justo antes de morir lo entrega a la logia a la cual había pertenecido su padre. Este hecho causa un gran revuelo y, después de unas peripecias, los masones deciden pasar estos "papeles de Heredia" a la familia de Lola Junco, el primer gran amor romántico del poeta. Esta familia pertenece a la flor y nata de la burguesía cubana y la publicación de este manuscrito podría causarles problemas porque se revelaría que un miembro suyo descendía del propio poeta. Al final, uno de los miembros de la familia decide destruir los papeles para deshacerse, de una vez por todas, de esta carga. Así que ahora podemos revelar que Fernando Terry nunca encontrará esos papeles.

La tercera trama narrativa (C) y quizá la más abundante es la autobiografía del propio poeta José María Heredia. Esta autobiografía son estos "papeles de Heredia" que quedan destruidos porque revelan muchos secretos. Entre ellos se encuentra también el de la fundación de la literatura cubana.

Estamos entonces ante tres tramas narrativas que alternan entre sí sin un orden matemático preestablecido, pero sí observan una cohesión interna, porque, al parecer, las secuencias que 
narran las tramas $\mathrm{B}$ y $\mathrm{C}$ intentan responder a las preguntas que se formulan Terry y sus amigos en A. En cuanto a los narradores estamos ante dos modalidades: un narrador homodiegético, que narra la autobiografía de Heredia, y un narrador heterodiegético, que está detrás de las peripecias tanto del manuscrito como las de Fernando Terry. Este uso de narradores no hace sino confirmar la predilección por las estructuras triangulares que manifestó Padura en una entrevista (Bivort, 2013). El triángulo se manifiesta también en la ambientación temporal de la novela, porque cada una de las tramas narrativas transcurren en otro momento histórico. Mientras que la $\mathrm{C}$ pertenece a principios del siglo XIX, la B nos habla de finales del siglo XIX y principios del XX, ya que según un expreso deseo del poeta, los papeles no se podrían publicar hasta cien años después de su muerte, y, por último, la A que nos lleva a Cuba de los años setenta (en recuerdos de Terry) y de los años noventa (en "realidad").

En cuanto al género del texto, hemos dicho que prácticamente estamos ante tres textos interdependientes, pero que gozan de una cierta autonomía. De todas maneras, forman un todo que podemos catalogar como una novela negra ${ }^{2}$. El carácter detectivesco del texto le permite a Padura presentar, de una manera amena y entretenedora, el planteamiento de los problemas, formulando preguntas y buscando respuestas. Este procedimiento es también muy característico de la literatura posmoderna relacionada con las indagaciones historiográficas y, a día de hoy, la novela negra se ha convertido en un mercado no solo en los países nórdicos sino también en España. Esta novela policíaca ofrece una estructura narrativa sólida ya que permite avanzar cuando los protagonistas aumentan la información sobre el manuscrito mediante indicios, entrevistas, descubrimientos fortuitos, etc. La tensión creada también mediante preguntas $(\mathrm{A})$ y respuestas $(\mathrm{B}, \mathrm{C})$ crea una especie de suspense que agiliza la lectura. Dicho sea de paso, que al lado de la labor detectivesca que protagoniza Terry en cuanto a los papeles de Heredia, hay otra -aunque más psicológica y menos vi-

2 El propio Padura dijo que "la más policíaca de mis novelas es $L a$ novela de mi vida” (Wieser, 2005). 
sible- que comprende la búsqueda del delator culpable del exilio de Terry.

Todo lo dicho sobre la novela no es sino una información complementaria de su rasgo principal: la metaficción historiográfica. Uno de los logros -o desastres- del posmodernismo consiste en el saber de que la verdad ha dejado de existir. De ahí que la literatura -o arte en su totalidad- haya arrebatado a la historiografía el papel de contar los hechos pasados. Si antes existía un discurso sobre lo que pasó, ahora existen al menos dos: una verdad histórica y una poética, siendo la última la que prevalece, creando, al fin y al cabo, una multitud de discursos que pueden ser complementarios o no. La situación se complica cuando nos damos cuenta de que somos capaces de mezclar estas verdades para construir una nueva ${ }^{3}$. Hay textos que cuentan lo que ocurrió de verdad en la historia sin que se haya cambiado una sola coma -podemos denominarlos "relatos reales"- pero la forma de elaboración de estos textos es literaria. Estamos entonces ante unas "novelas sin ficción" que cuentan hechos históricos pero lo hacen mediante literatura. Cuando Hutcheon inaugura en 1988 el término "metaficción historiográfica", habla de los textos que representan el pasado histórico adquiriendo un carácter parecido al del discurso historiográfico pero sin perder su condición ficcional. Además, hay que destacar que en la metaficción historiográfica el trabajo se hace desde el presente lo que permite crear muchas interpretaciones, por lo cual el discurso ya no es único sino múltiple y el proceso de la creación de la memoria colectiva consiste en un constante reajuste de datos. Ahora bien, ¿̇si Padura dice que para escribir La novela de mi vida se basaba en una documentación rigurosa debemos comprender la trama $\mathrm{C}$ como un documento historiográfico o como un texto de ficción? Heredia dicta sus memorias antes de morir, en su lecho de muerte. El lector, al leerlas, tiene que acogerse al pacto autobiográfico ${ }^{4}$ pero el propio Heredia se refiere al texto que está dictando como a una novela, es decir un texto

3 Javier Cercas habla de la tercera verdad. Cfr. su artículo titulado "La tercera verdad", publicado en El País en 2011.

4 Concepto introducido por Phillippe Lejeune en 1975. 
puramente ficcional. Y si añadimos la información proporcionada por Padura en los peritextos, la duda sobre el carácter del texto que leemos no queda resuelta hasta el último momento, cuando el lector se entera de que los papeles de Heredia fueron destruidos (¿Pero fueron destruidos de verdad o solamente en la ficción?).

Una de las lecturas que permite la novela consta entonces de un repensamiento del propio pasado cubano. En virtud de las teorías de la nueva novela historiográfica y las de la memoria histórica podemos decir que un eje, alrededor del cual gira la obra, es la cubanía. Es precisamente la época del Romanticismo cuando en Europa surgen naciones en el sentido moderno de la palabra y es, al mismo tiempo, cuando en América se emancipan sociedades que pretenden formar países. Sin duda alguna, había cierta identificación protonacional de los americanos con el territorio y la sociedad local, pero para poder hablar de las naciones se necesitaba algo que podemos llamar "ficciones fundacionales". La búsqueda de tal mito y el debate sobre la identidad se llevaron a cabo también en Cuba, aunque con un considerable retraso. Es precisamente la estética romántica que dio al mundo al primer poeta cubano José María Heredia. Él y un grupo de sus amigos participaban activamente en la lucha pro-independentista de Cuba a principios del siglo XIX. Heredia entró en la logia masónica y preparaba una sublevación, lo que le valió un destierro de casi toda la vida. No obstante, lo que no pudo hacer con armas lo hizo con la pluma y su poesía se convirtió en una de las voces más agudas que gritaban por la libertad de la isla. José Martí consideraba a Heredia fundador de la literatura cubana, cuyos versos, por primera vez en la historia, expresaban la quintaesencia de lo cubano. José María Heredia podría representar entonces este mito fundacional. Sin embargo, cuando abrimos cualquier antología de la poesía cubana ${ }^{5}$ aparece como el verdadero fundador un escribano canario Silvestre de Balboa que escribió en 1608 un poema titulado "Espejo de paciencia". Este poema es el primer texto conocido sobre Cuba encontrado al azar por el escritor José Antonio

5 Cfr. e.g. J. Lezama Lima (2002), Antología de la poesía cubana. Tomo I (Siglos XVII-XVIII), Madrid: Verbum. 
Echeverría en 1836. En los papeles de Heredia (C) se nos cuenta, no obstante, que todo este poema es un fraude preparado por los antiguos amigos de Heredia quienes le envidiaban su fama. Se trataba, sobre todo, del amigo íntimo de Heredia, Domingo del Monte, un importante crítico y escritor cubano. Al inventar el poema se llegaría a tener una excelente ficción fundacional que procediera de las mismas fechas que las primeras obras mexicanas o peruanas ${ }^{6}$. Al hablar de este episodio, no deberíamos olvidar la profesión original de Fernando Terry: profesor universitario que escribió su tesis sobre Heredia y que, por lo tanto, posee una sólida preparación filológica. La importancia que se da a la labor de humanidades en la construcción de relatos nacionales emana de todo el libro. Otra vez, este juego a caballo entre la ficción y la realidad nos puede parecer una buena invención del escritor maestro como Padura, pero si en la literatura checa pasó algo parecido a principios del siglo XIX, ¿qué pensar ahora ${ }^{7}$ ?

Es evidente que los juegos con los hechos históricos permiten sacar conclusiones contradictorias, mirar los hechos históricos desde diferentes ángulos o hasta negar su propia existencia. En cuanto a La novela de mi vida falta este carácter reivindicativo que encontramos en la narrativa de la memoria histórica en España

6 A modo de añadidura podemos decir que el debate se prolongó hasta muy entrado el siglo XX cuando los intelectuales debatían sobre el origen de la cultura cubana disputando su primacía los negros y los indígenas. Cfr. J. Mlčoch (2014), "Algunas consideraciones acerca de la música en Cuba y su influencia en la obra de Alejo Carpentier", en T. Jaromin, M. Kolankowska, P. Sawicki (eds.), Traducir una cultura a otra, Wrocław: Wydawnictwo Wyższej Szkoły Filologicznej we Wrocławiu, 11-17.

7 En la segunda década del siglo XIX se descubrieron por azar en Praga y en Zelená Hora dos textos que databan supuestamente del siglo XIII. Debido a que la literatura checa carece de grandes poemas épicos medievales estos textos, en principio, debieron cumplir esta función. Nada más aparecer se desató una polémica sobre su veracidad. Al final en los años sesenta del siglo pasado se demostró que se trataba de un fraude orquestado por V. Hanka, J. Linda y F. Horčička. 
que llega hasta límites fraudulentos, pero sí podemos observar la singular capacidad de Padura con la cual une la ficción con la historia, formulando así varias preguntas sobre la identidad cubana. José María Heredia es tomado como modelo de la cubanía y las peripecias vitales que nos describe en su lecho de muerte se parecen bastante a las que vive el protagonista de la trama narrativa A. Esto nos permite observar cierta identificación entre los dos protagonistas. Ahora bien, esta identificación puede interpretarse de dos maneras: por un lado, estamos ante una clara continuidad, tal y como indica Ángel Esteban (2006). El carácter cíclico de la novela -y por extensión del universo- es, sin duda alguna, un rasgo definitorio de América Latina. Terry es Heredia, los dos sufren los mismos problemas, los dos sienten la misma felicidad, los dos son una esencia de los cubanos quienes tienen que luchar siempre contra las adversidades impuestas por un poder ajeno. Pero, por otro lado, podemos encontrar una interpretación desmitificadora. La cubanía no es una lucha contra los poderes ajenos que no permiten el desarrollo -fíjense en la similitud de esta afirmación con el discurso oficial del gobierno castrista- sino todo lo contrario. La cubanía puede interpretarse como un continuo fracaso causado no por los poderes ajenos sino por la incapacidad de los propios cubanos. No fueron los "yankees" quienes impidieron a Heredia lograr la independencia de Cuba, sino los propios cubanos que temían que la independencia pudiera dar lugar a las sublevaciones de los esclavos, parecidas a Haití. Tampoco Terry tuvo éxito en la búsqueda tanto de los papeles de Heredia como en la de su supuesto delator. Su fracaso se ve aumentado también por sus circunstancias vitales: un exiliado que tuvo que huir de la isla por la dictadura, no impuesta desde el extranjero, sino creada desde dentro de la nación. Al igual que Heredia es un mito fundacional de la cubanía, Terry es la cubanía actual, visiblemente dolorida, traumatizada y dividida por la dictadura castrista.

El libro se cierra con las siguientes reflexiones que demuestran, siguiendo los preceptos teóricos antes expuestos, la situación indecisa, caótica, nostálgica e impredecible de toda la sociedad cubana oprimida por la dictadura. 
Fernando mira a sus amigos y piensa que quizás el Varo no merecía ser aquel alcohólico empedernido, ya sin capacidad de escribir poesía, o que el Negro podía haber sido para siempre un eterno y nada problemático creyente, quizá sólo uno de esos personajes que pasan levemente por la vida sin mirar hacia los lados y sin saber siquiera el color de su piel. El exceso de cinismo de Tomás le parece un ensañamiento con él, mientras al guajiro Conrado lo han moldeado como un lépero demasiado evidente, despojado - con toda intención- de la inocencia tradicional en los tipos de su especie y origen. Incluso la fe en la poesía de Arcadio le resultaba desproporcionada, a pesar de vivir en una historia de poetas, pues ya nadie practica aquella mística pasada de moda [...]

¿Siempre habrá sido así?, se pregunta entonces, al recordar las veleidades del destino de José María Heredia, arrastrado por los flujos y reflujos de la historia, el poder y ambición, atrapado en un torbellino tan compacto que lo llevó a sentir, con apenas veinte años, el signo novelesco que marcaba su existencia. ¿Es posible rebelarse?, se pregunta después, ya por pura retórica, sólo para abrir más la herida, pues sabe que el acto de la rebeldía es el primero que les ha sido negado, radicalmente extirpado de todas sus posibilidades y anhelos. Sólo le queda cumplir su moira, como Ulises enfrentó la suya, aun a su pesar; o como Heredia asumió la suya, hasta el final [...] (Padura, 2002a: 342).

Para concluir, podemos decir que la lectura de La novela de mi vida provoca en el lector una sensación de inseguridad. Los "papeles de Heredia" fueron destruidos en la novela, pero el mal sabor de boca se queda. Hay demasiadas preguntas que deja Padura sin resolver. ¿Pero, acaso, no se trata precisamente de eso? ¿No es este el papel de la literatura de la memoria? ¿Intentar postular preguntas que deberían responder luego los lectores (o los historiadores) en vez de usurpar para sí la única verdad? 


\section{Bibliografía}

Bivort, S. (2013). "Leonardo Padura y la dignidad del derrotado". Letral, 10, 152-158.

Carpentier, A. (2003). "La novela latinoamericana en vísperas de un nuevo siglo". Ensayos selectos. Buenos Aires: Ediciones Corregidor.

Cercas, J. (2011). “La tercera verdad”. El País, 25 de junio de 2011, [en línea] <https:/elpais.com/diario/2011/06/25/babelia/1308960747_850215.html> [28.04.2018].

Esteban, Á. (2006). "Heredia que se repite: La isla y los tiranos", en: Á. Esteban (ed.) Literatura cubana entre el viejo y el mar, 316-335. Granada: Renacimiento.

González Echevarría, R. (2011). Mito y Archivo: una teoría de la narrativa latinoamericana. México: Fondo de cultura económica.

Hutcheon, L. (2014). Una poética del postmodernismo. Buenos Aires: Prometeo.

Lejeune, P. (1975). Le pacte autobiographique. París: Ed. Seuil.

Lezama Lima, J. (2002). Antología de la poesía cubana. Tomo I (Siglos XVII-XVIII). Madrid: Verbum.

Mlčoch, J. (2014). "Algunas consideraciones acerca de la música en Cuba y su influencia en la obra de Alejo Carpentier", en: T. Jaromin, M. Kolankowska, P. Sawicki (eds.). Traducir una cultura a otra, 11-17. Wrocław: Wydawnictwo Wyższej Szkoły Filologicznej we Wrocławiu.

Viu, A. (2007). "Una poética para el encuentro entre la realidad y la ficción”. Revista chilena de literatura, 70, 167-178.

Wieser, D. (2005). "Leonardo Padura: «Siempre me he visto como uno más de los autores cubanos»", Espéculo, 29, [en línea] $<$ https://webs.ucm.es/info/especulo/numero29/padura.html> [28.04.2018]. 